\title{
Efficacy of Fentanyl Transdermal Patch in the Treatment of Chronic Soft Tissue Cancer Pain
}

\author{
Karim Hemati ${ }^{1}$; Behrooz Zaman ${ }^{2,}$; Valliolah Hassani ${ }^{3}$; Farnad Imani ${ }^{3}$; Parviz Dariaie ${ }^{4}$ \\ ${ }_{1}^{1}$ Department of Anesthesiology, Imam Khomieni Teaching Hospital, Ilam University of Medical Sciences, Ilam, Iran \\ 2 Department of Anesthesiology, Hazrat Fatemeh Teaching Hospital, Iran University of Medical Sciences, Tehran, Iran \\ 3 Department Of Anesthesiology, Rasool Akram Teachig Hospital, Iran University Of Medical Sciences, Tehran, Iran \\ ${ }^{4}$ Imam Khomeini Teaching Hospital, Tehran University of Medical Sciences, Tehran, Iran \\ *Corresponding author: Behrooz Zaman, Department of Anesthesiology, Fatemeh Zahra Teaching Hospital, Iran University of Medical Sciences, Tehran, Iran. Tel: +98-9123047764, Fax: \\ +98-2166509059, E-mail: bhrzzaman@gmail.com
}

Received: August 19, 2014; Revised: October 9, 2014; Accepted: October 12, 2014

\begin{abstract}
Background:Cancer pain may be a major problem for health care providers worldwide. According to different studies reporting the pain severity, one-third of patients reported to have moderate to severe pain. Management of cancer pain is one of the most important goals of palliative care. Recently, different research results on the efficacy of opioid analgesics in chronic pain management have played a role to implement standards in pain control by government agencies worldwide.

Objectives: This study aimed to investigate the efficacy of fentanyl transdermal patch in the treatment of chronic soft tissue cancer pain. Patients and Methods: In a prospective descriptive study, we evaluated 86 patients with soft tissue tumors with chronic pain referred to cancer institute of Imam Khomeini Hospital, Tehran, Iran, during 2006-2007. For all patients, transdermal fentanyl patch (25 $\mu \mathrm{g} / \mathrm{h}) \mathrm{was}$ administered. The appearance of patches was the same. Pain severity was measured by Visual Analogue Scale (VAS) initially and 24,48 and 72 hours after the initiation of treatment.

Results: Patients' characteristics and VAS score before the treatment were not significantly different $(\mathrm{P}>0.05)$. According to our findings, the pain severity was significantly reduced after the treatment $(\mathrm{P}=0.001)$. The incidence of adverse events in patients was significantly high (72\%). The most common adverse events were sleepiness, nausea and vomiting in $30.2 \%$ and $18.6 \%$, respectively.

Conclusions:Transdermal fentanyl patch was an effective and safe method to reduce pain in patients with soft tissue tumors. Moreover, it could improve the quality of life in these patients, but adverse events occurred in approximately $72 \%$ of patients.
\end{abstract}

Keywords:Chronic Pain; Fentanyl Transdermal Patch; Soft Tissue Malignancy

\section{Background}

Today in Iran, cancer is an important challenge to health care systems $(1,2)$. According to the annual report of the World Health Organization, the incidence of cancer was 12667470 new cases in 2008 and estimated to be over 15 million in 2020. Therefore, cancer pain may be a major problem for health care providers worldwide (3). According to different studies reporting the pain severity, one-third of patients reported to have moderate to severe pain (3). In patients with cancer pain, transdermal fentanyl patch could be an efficient and safe long-term analgesic method. There are four different forms of fentanyl transdermal patches 25, 50, 75 and $100 \mathrm{mcg} / \mathrm{hour}$. The transdermal patch releases fentanyl at a constant rate for up to 72 hours. Fentanyl is a synthetic opioid with short-acting analgesic activity. The fentanyl patch is a proper option for cancer pain control. However, one of the most encountered problems is pain control deterioration because of decreased fentanyl release from the patch on the third day of treatment before patch replacement. It was reported that this complication would be seen in about one quarter of cases (3). The efficacy and onset of action of transdermal patches depend on many factors including the site of attachment, local and more generalized warmness and skin damage or abrasions. To minimize these conflicting effects, it has been suggested to: 1) patches should be applied to non-damaged skin of the deltoid muscle, lateral and dorsal thorax; 2) the area should be scraped and cleaned with water; and 3) no external heat application to the patch site (3). Fentanyl is a known opioid with unique property including a more potent opioid ( $\sim 75$ times more than morphine), a smaller molecular mass, proper lipid solubility and greater transdermal permeability than morphine. These properties make it suitable for transdermal administration. Every fentanyl transdermal patch provides constant blood concentration of fentanyl for 72 hours. The blood concentration rises slowly and these properties reduce the risk of side effects. Fentanyl patch metabolites are not pharmacologically active and not affected by the firstpass effect in the liver or by gastrointestinal absorption. Furthermore, fentanyl has higher selectivity and affinity for $\mu 1$ receptors. Therefore, there is no side effect caused

Copyright (C) 2015, Iranian Society of Regional Anesthesia and Pain Medicine(ISRAPM). This is an open-access article distributed under the terms of the Creative Commons Attribution-NonCommercial 4.0 International License (http://creativecommons.org/licenses/by-nc/4.0/) which permits copy and redistribute the material just in noncommercial usages, provided the original work is properly cited. 
by $\mu 2$ receptors activation such as nausea, vomiting and constipation seen with morphine. In addition, there are no other side effects related to accumulation of metabolites.

Management of cancer pain is one of the most important goals of palliative care (4). Recently, different research results on the efficacy of opioid analgesics in chronic pain management played a role to implement standards in pain control worldwide (5). Pain is a highly prevalent and distressing symptom and a major health problem in patients with cancer (6). The pain incidence among patients with cancer ranges from $14-100 \%$ and occurs in $50-70 \%$ of patients in active phase of treatment. According to the literature reports, pain would be seen in as high as $60-90 \%$ of patients with advanced stages of cancer (7-9).

Most patients with cancer may require the use of opioid during the course of their illness. Cancer pain control can be achieved adequately in most patients with oral analgesics. However, in some instances, pain may not respond to optimal dosages of opioid. The main reason for this problem is the adverse effects before achieving a favorable analgesia or poor analgesic response despite rapid dosage escalation. Opioid drug change may be needed to identify the proper drug that yields the most suitable balance between analgesia and adverse effects. In some patients, side effects such as generalized nausea, vomiting, myoclonus, delirium or severe sedation may be developed before achieving adequate analgesia (10).

There have been many clinical trials on the efficacy and side effects of transdermal fentanyl patches for cancer and non-cancer pain control $(11,12)$. Compared with other opioids, fentanyl patches have been associated with better pain control, less side effects such as constipation, nausea, vomiting, myoclonus, delirium and somnolence. This drug promotes the quality of life $(13,14)$. The use of fentanyl patch, a potent opioid with continuous release of drug, provided new options and its benefit is evident for patients unable to take oral medications. Therapeutic safety of transdermal fentanyl varies widely among patients. Fentanyl serum concentrations increase gradually after initial application, and peak serum concentrations of fentanyl generally reached 12-48 hours after the initial application. As previously mentioned, we might encounter difficulties for adjusting the conversion (10).

The challenges of symptom associated with cancer pain make it difficult to implement high quality trials. Significant technical flaws have been recognized, including small trial size, lack of uniform scale of pain, as well as variability in the definition of statistically significant "pain relief". In addition, there are no comparisons between opioids and other interventions, adjuvant medications, injections and blocks (6). In spite of increased knowledge of pain and its treatment in the recent years, treating pain of patients with cancer is often inadequate and results of fentanyl efficacy are controversial

\section{Objectives}

The aim of this study was to explore the effect of transdermal fentanyl patch in the treatment of chronic soft tissue cancer pain in a clinical trial of patients admitted to cancer institute, Imam Khomeini hospital, Tehran, Iran.

\section{Patients and Methods}

\subsection{Study Design}

The study design was a prospective descriptive study conducted from December 2006 to December 2007 at cancer institute, Imam Khomeini hospital, Tehran, Iran.

\subsection{Setting}

The study was confirmed by the research deputy of faculty of medicine, Tehran University of Medical Sciences. A written informed consent was obtained from patients.

\subsection{Selection of Participants}

Eighty-six patients with soft tissue tumors and chronic pain admitted to different wards of cancer institute, Imam Khomeini hospital, Tehran, Iran, were enrolled in the study. Inclusion criteria were age $>18$, life expectancy $>3$ months, assessable pain intensity and Visual Analogue Scale $(V A S)>3$. The exclusion criteria were allergy to opioid, a history of opioid use, bilirubin $\geq 2 \mathrm{~g} /$ $\mathrm{dL}$, creatinine level $\geq 2.0 \mathrm{mg} / \mathrm{dL}$, active skin disease, critical respiratory disorders, asthma, fever above $40^{\circ} \mathrm{C}$, impaired level of consciousness, $\mathrm{Co}_{2}$ retention, a history of radiotherapy, hormonal change and a history of chemotherapy in the last seven days. The study was approved by the University of Tehran Ethics Committee, and informed consent was obtained from all patients. All patients were fully informed about the procedure and the purpose of the experiment.

\subsection{Intervention}

Eighty-six patients with soft tissue cancer pain were included. Patients were unaware of the kind of drug administered. The pain severity was assessed before and after the treatment at different time intervals of 24,48 and 72 hours. Standard VAS scale was used to evaluate the pain severity from zero to 10 . No pain was considered as zero and the maximum pain ever experienced as 10 . A general examination was performed by physicians after recording clinical and demographic characteristics. For all patients, transdermal fentanyl patch $(25 \mu \mathrm{g} / \mathrm{h})$ was administered. The patches were applied to flat areas of deltoid and replaced every 72 hours. We used a patch for 72 hours and only in five cases due to getting wet patch, a new patch was used. To evaluate the analgesic efficacy of patch, the degree of pain was assessed daily in the morning. The pattern of sleep was assessed daily using a qualitative five-point scale of "very well," "well," "normal," "poor," and "very poor. 
Hemati Ket al.

\subsection{Methods of Measurement}

The safety and effectiveness of treatment was evaluated in the first day every eight hours and the next days once a day measuring blood pressure, pulse rate, respiratory rate and temperature. In the case of blood pressure decrease, bradycardia or loss of consciousness, we initiated supportive care and if the vital sign instability continued, patch was removed. Besides, the severity of side effects such as nausea, vomiting, sleepiness, constipation and skin changes was evaluated. Additional dosage of opioids or non-opioids and other drug treatment were documented.

\subsection{Outcome Measures}

Primary outcome was subsiding cancer pain in the first 12-18 hours after the initiation of treatment. The secondary objective was evaluating pain score in the next three days.

\subsection{Primary Data Analysis}

All statistical analyses were performed using SPSS version 13.0 (SPSS Inc., Chicago, IL). Categorical data were expressed as percentages and comparisons were made using $\chi^{2}$ tests. One-way ANOVA and independent t-test were used to compare parametric scores. $\mathrm{P}<0.05$ was considered as statistically significant. Values were presented as mean \pm SD.

\section{Results}

Baseline patients' characteristics are shown in Table 1. None of 86 enrolled patients withdrew from the study. The VAS score before the treatment was not significantly different between the patients $(\mathrm{P}>0.05)$.

\subsection{Main Result}

Patients who received transdermal fentanyl patch had a significantly lower pain in 24 hours, 48 hours and 72 hours after the treatment $(P=0.001)$ (Table 2). The incidence of adverse events in transdermal fentanyl patch group was significantly high (72\%). sleepiness in 26 (30.2\%), Nausea and vomiting in 16 patients (18.6\%) constipation in 16 patients (18.6\%), pruritus in 2 patients (2.3\%) and redness (Local redness ) in 2 patients (2.3\%) were observed (Table 2 ).

\section{Discussion}

Fentanyl transdermal patch was approved by the FDA in 1990 and now used in more than 50 countries worldwide (15). This was the first clinical trial to evaluate the efficacy of transdermal fentanyl patch for the treatment of chronic soft tissue cancer pain in an Iranian population. Our result showed that the use of transdermal fentanyl patch significantly decreased the severity of pain in patients with cancer. This finding is consistent with some other studies (11-17). Fentanyl patch was reported to have fewer adverse events ( $2 \%$ to $30 \%$ ) and the most frequently mentioned adverse events were nausea, vomiting and constipation (16-20). In other studies, adverse events were reported $72 \%$ in transdermal fentanyl patch (21). The most frequently mentioned adverse events were nausea, vomiting and sleepiness (22) and pain intensity remained stable and adverse events occurred in $20 \%$ of patients in 12 hour method (22). The studies support the effectiveness of fentanyl transdermal patch for treating cancer and non-cancer pain (23-28). In a systematic review of opioids consumption for cancer pain, fair evidence for the efficacy of transdermal fentanyl and poor evidence for morphine, tramadol, oxycodone, methadone and codeine were reported (29). There is a biopsychosocial experience with a significant cognitive and emotional component with cancer pain. Patients with anxiety and depression and cancer express higher levels of pain. There is a strong correlation between daily high-level distress and reporting high levels of pain (29).

\begin{tabular}{lc}
\hline Table 1. Demographics and Baseline Characteristics of Patients ${ }^{\mathrm{a}}$ \\
\hline Variables & Fentanyl Group $(\mathbf{n}=\mathbf{8 6})$ \\
\hline Age, $\mathbf{y}$ & $45.5 \pm 18$ \\
Gender, male/female & $48 / 38$ \\
\hline Site of tumor & \\
\hline Extremities & $38(44.1)$ \\
\hline Retroperitoneal & $26(32.5)$ \\
Trunk and chest & $8(9.3)$ \\
\hline Head and neck & $12(13.9)$ \\
\hline Mediastinum & $2(2.3)$ \\
\hline Duration of pain, mo & $14.3 \pm 20.2$ \\
\hline a Data are presented as mean \pm SD or No. (\%). &
\end{tabular}

Table 2. Outcome Parameters $(n=86)^{a}$

\begin{tabular}{lcc}
\hline Outcome Parameters & Fentanyl (After) & P Value \\
\hline VAS score before the treatment & $6.2 \pm 0.7$ & $>0.05$ \\
$\begin{array}{l}\text { VAS score after } 24 \text { hours of the } \\
\text { treatment }\end{array}$ & $3.5 \pm 0.8$ & 0.001 \\
$\begin{array}{l}\text { VAS score after } \mathbf{4 8} \text { hours of the } \\
\text { treatment }\end{array}$ & $3.4 \pm 0.7$ & 0.001 \\
$\begin{array}{l}\text { VAS score after 72 hours of the } \\
\text { treatment }\end{array}$ & $3.4 \pm 0.7$ & 0.001 \\
$\begin{array}{l}\text { Number of side effect after the } \\
\text { treatment }\end{array}$ & $24(27.9)$ & - \\
$\begin{array}{l}\text { Sleepiness } \\
\text { Nausea and vomiting }\end{array}$ & $26(30.2)$ & 0.001 \\
Constipation & $16(18.6)$ & 0.001 \\
\hline $\begin{array}{l}\text { Redness } \\
\text { Pruritus }\end{array}$ & $16(18.6)$ & 0.001 \\
\hline
\end{tabular}

\footnotetext{
${ }^{\mathrm{a}}$ Data are presented as mean \pm SD. or No. (\%).
} 
However some recommendations are required for the safety of patients; cautious administration and close medical observations, in the first week daily contact by phone, and after that monthly visit in the outpatient setting (29). Sleepiness in transdermal fentanyl patch was one of the problems in patients. If it was possible to measure actual serum fentanyl level, the effective dosage with fewer adverse events was delivered. Further studies are needed to measure the actual serum fentanyl concentrations.

To conclude, fentanyl transdermal patch could provide stable pain control and removing the need for injections, thereby enhancing patient's quality of life. Fentanyl transdermal patch $(25 \mu \mathrm{g} /$ hour $)$ provided an effective treatment for cancer pain, but adverse events occurred in approximately $72 \%$ of patients. More educational programs on appropriate use of fentanyl transdermal patch for cancer pain management are required in Iran.

This study was conducted on a limited number of patients with soft tissue tumor. This was a limitation of the present study. This study could also be conducted on multiple cancer departments. Therefore, we recommend a new research with greater sample size in some sessions. Another limitation was not measuring the serum fentanyl concentration. Sleepiness was one of the adverse effects in patients. An effective dosage with fewer adverse events could be administered if serum fentanyl level was measured. Therefore, it is suggested to perform further studies to measure serum fentanyl concentrations.

\section{Acknowledgements}

We thank participants, coordinators and data reviewers who assisted in this study.

\section{Authors' Contributions}

Dr. Karim Hemati and Dr. Behrooz Zaman: study concept and design revision. Dr. Valliolah Hassani and Dr. Farnad Imani: statistical analysis. Dr. Parviz Dariaie: interpretation of data.

\section{Funding/Support}

This study was supported by Iran University of Medical Sciences (4275).

\section{References}

1. Ghavamzadeh A, Moussavi A, Jahani M, Rastegarpanah M, Iravani M. Esophageal cancer in Iran. Semin Oncol. 2001;28(2):153-7.

2. Montazeri A, Sajadian A, Ebrahimi M, Haghighat S, Harirchi I. Factors predicting the use of complementary and alternative therapies among cancer patients in Iran. Eur J Cancer Care (Engl). 2007;16(2):144-9.

3. Ripamonti CI. Pain management. Ann Oncol. 2012;23 Suppl 10:x294-301.

4. Kim JH, Kim M, Sung CW, Kim HS, Jang HJ, Shin YC, et al. Highdose fentanyl patch for cancer pain of a patient with cholangiocarcinoma. Korean J Intern Med. 2010;25(3):337-40.

5. Park JH, Kim JH, Yun SC, Roh SW, Rhim SC, Kim CJ, et al. Evaluation of efficacy and safety of fentanyl transdermal patch (Durogesic D-TRANS) in chronic pain. Acta Neurochir (Wien). 2011;153(1):18190.

6. Koyyalagunta D, Bruera E, Solanki DR, Nouri KH, Burton AW, Toro MP, et al. A systematic review of randomized trials on the effectiveness of opioids for cancer pain. Pain Physician. 2012;15(3 Suppl):ES39-58.

7. Christo PJ, Mazloomdoost D. Cancer pain and analgesia. Ann N Y Acad Sci. 2008;1138:278-98.

8. Keefe FJ, Abernethy AP, C. Campbell L . Psychological approaches to understanding and treating disease-related pain. Annu Rev Psychol. 2005;56:601-30.

9. Esmo Guidelines Working Group , Jost L. Management of cancer pain: ESMO clinical recommendations. Ann Oncol. 2007;18 Suppl 2:ii92-4.

10. Nomura M, Kamata M, Kojima H, Hayashi K, Kozai M, Sawada S Six- versus 12-h conversion method from intravenous to transdermal fentanyl in chronic cancer pain: a randomized study. Support Care Cancer. 2011;19(5):691-5.

11. Donner B, Zenz M, Strumpf M, Raber M. Long-term treatment of cancer pain with transdermal fentanyl. J Pain Symptom Manage. 1998;15(3):168-75.

12. Grond S, Zech D, Lehmann KA, Radbruch L, Breitenbach H, Hertel D. Transdermal fentanyl in the long-term treatment of cancer pain: a prospective study of 50 patients with advanced cancer of the gastrointestinal tract or the head and neck region. Pain. 1997;69(1-2):191-8.

13. Radbruch L, Sabatowski R, Loick G, Kulbe C, Kasper M, Grond S et al. Constipation and the use of laxatives: a comparison between transdermal fentanyl and oral morphine. Palliat Med. 2000;14(2):111-9.

14. Mercadante S. Opioid rotation for cancer pain: rationale and clinical aspects. Cancer. 1999;86(9):1856-66.

15. Hoya Y, Okamoto T, Yanaga K. Evaluation of analgesic effect and safety of fentanyl transdermal patch for cancer pain as the first line. Support Care Cancer. 2010;18(6):761-4.

16. Choi HS, Kim KO, Chun HJ, Keum B, Seo YS, Kim YS, et al. The ef ficacy of transdermal fentanyl for pain relief after endoscopic submucosal dissection: a prospective, randomised controlled trial. Dig Liver Dis. 2012;44(11):925-9.

17. Komurcu S, Turhal S, Altundag K, Atahan L, Turna HS, Manavoglu $\mathrm{O}$, et al. Safety and efficacy of transdermal fentanyl in patients with cancer pain: phase IV, Turkish oncology group trial. Eur J Cancer Care (Engl). 2007;16(1):67-73.

18. Calis KA, Kohler DR, Corso DM. Transdermally administered fentanyl for pain management. Clin Pharm.1992;11(1):22-36.

19. Konishi H, Ishihara C, Chiba M, Endo Y, Yamaji A. Pain recurrence on the third day after application of a transdermal fentanyl patch. Pharm World Sci. 2005;27(4):353.

20. Kanamori C, Kanamori T, Hayashi M, Yorioka H, Kanzaki H. Threecycle fentanyl patch system significantly improves pain control in gynecologic cancer.J Obstet Gynaecol Res. 2006;32(6):605-9.

21. Kanamori C, Kanamori T, Tanaka Y, Kanzaki H. Three-cycle fentanyl patch system contributes to stable control of plasma fentanyl concentration in gynecologic cancer pain patients. Taiwan $\mathrm{J} \mathrm{Ob}$ stet Gynecol. 2011;50(1):79-84.

22. Kornick CA, Santiago-Palma J, Khojainova N, Primavera LH, Payne R, Manfredi PL. A safe and effective method for converting cancer patients from intravenous to transdermal fentanyl. Cancer. 2001;92(12):3056-61.

23. Radbruch L, Sabatowski R, Petzke F, Brunsch-Radbruch A, Grond S, Lehmann KA. Transdermal fentanyl for the management of cancer pain: a survey of 1005 patients. Palliat Med. 2001;15(4):309-21.

24. Cachia E, Ahmedzai SH. Transdermal opioids for cancer pain. Curr Opin Support Palliat Care. 2011;5(1):15-9.

25. Skaer TL. Transdermal opioids for cancer pain. Health Qual Life Outcomes. 2006;4:24.

26. Hoy SM, Keating GM. Fentanyl transdermal matrix patch (Durotep MT patch; Durogesic DTrans; Durogesic SMAT): in adults with cancer-related pain. Drugs. 2008;68(12):1711-21.

27. Ruoff GE, Rosenthal N, Jordan D, Karim R, Kamin MProtocol, 


\section{Hemati Ket al.}

Capss-Study Group. Tramadol/acetaminophen combination tablets for the treatment of chronic lower back pain: a multicenter, randomized, double-blind, placebo-controlled outpatient study. Clin Ther. 2003;25(4):1123-41.

28. Peloso PM, Fortin L, Beaulieu A, Kamin M, Rosenthal N, Protocol, T. R. P. C. A. N. Study Group . Analgesic efficacy and safety of tramadol/ acetaminophen combination tablets (Ultracet) in treatment of chronic low back pain: a multicenter, outpatient, randomized, double blind, placebo controlled trial.J Rheumatol. 2004;31(12):2454-63.

29. Reed F, Burrow R, Poels KL, Godderis L, Veulemans HA, Mosing M. Evaluation of transdermal fentanyl patch attachment in dogs and analysis of residual fentanyl content following removal. Vet Anaesth Analg. 2011;38(4):407-12. 